\title{
CSE without Math? A First Course in Modeling and Simulation
}

\author{
Wolfgang Wiechert \\ Department of Simulation, Faculty of Mechanical Engineering, \\ University of Siegen, Germany \\ wiechert@simtec.mb.uni-siegen.de, \\ http://www.simtec.mb.uni-siegen.de
}

\begin{abstract}
There are several good reasons to accompany a course in computational science and engineering from the very beginning with a general lecture on modeling and simulation. On the other hand first year undergraduate students only have elementary knowledge on mathematics and natural sciences. Consequently, a first lecture must be conceived that requires only basic mathematical and physical skills. The course on 'Foundations of Modeling and Simulation' given at the university of Siegen fulfills this requirements. It is the first in a series of 7 lectures on 'Modeling and Simulation' which later on will go much deeper into the technical details. Basic didactic considerations, examples and experiences from the first course are presented. In particular an extensive example of a fairground looping swing is discussed.
\end{abstract}

\section{Introduction}

No course in computational science and engineering (CSE) or in modeling and simulation (M\&S) can be given without a substantial amount of mathematical contents. For that reason teaching CSE without mathematics seems to be a contradiction. However, there are some good reasons to start education in CSE with an introductory course in which mathematical subjects have been strongly reduced:

Virtual and real world: Simulation projects require a close cooperation of researchers from different disciplines. The steps that frequently are the most critical for the success of a simulation project are project definition, model validation and communication of the results (cf. Fig. 1). These are exactly those steps where the virtual world meets reality. To sensitize the students for the 
problems arising at this interface they should be led to their first practical simulation project as quickly as possible. In particular the aspect of communication in multi disciplinary teams, the specification of the right project goals and a reasonable balance between algorithmic performance and overall time to deliver the results must be taught.

Multi disciplinarity: Due to its multi disciplinary nature CSE covers a wide range of methods from mathematics and computer science to the applications in the natural sciences and engineering. For a student it is hard to survey all this different subjects, their connections and interdependencies. Consequently, the students need to learn very early how to integrate subjects from different disciplines in the focus of a certain application problem. Thus example projects which require little in depth knowledge of mathematics, computer science and natural sciences should accompany a CSE course from the very beginning.

Heterogeneous audience: There are only a few universities were a complete CSE program with undergraduate and graduate courses is established. A good alternative is to establish CSE as a specialization in classical study programs like engineering, mathematics or physics or to establish a graduate course which can be attended with different prerequisites. In each case it is a big problem to integrate all the students with their different origins and to synchronize their knowledge in the basic disciplines. In this situation a first course on M\&S should introduce into the subject without relying on advanced skills in mathematics, computer science and physics. Accompanying simulation projects will give the students the opportunity to profit from their different skills.

CSE in practice: Not only at the university but also in industry CSE methods have become an important part of research and development processes. Project managers in industry know that simulation is a potential tool to understand complex systems, to evaluate experiments, to build virtual prototypes or to judge the potential economic benefit of a development. On the other hand this creates a need for a basic M\&S education even for non CSE specialists. Group leaders and managers should at least be able to judge under which conditions simulation may be a useful tool, what the basic problems are and what the costs of a simulation project will be.

\section{Simulation Courses at the University of Siegen}

\subsection{Different Prerequisites in a Heterogeneous Audience}

The Department of Simulation at the University of Siegen is a part of the engineering faculties (mechanical and electrical engineering, computer science). The lectures on M\&S are attended by students with quite different preconditions taking part in different study programs:

1. M\&S can be chosen as an optional course for all students of electrical and mechanic engineering. Both have basic mathematical skills from undergraduate courses and also a good knowledge of the physics of mechanical and electrical systems. On the other hand engineers are typically less trained in computer science and programming. 
2. These prerequisites are quite different for the computer science students. Although they also have several mathematical lectures in the undergraduate courses they are usually not familiar with the basics of analysis, numerical algorithms and differential equations. Moreover, it requires a high effort for them to understand the laws of physics.

3. Lectures on $M \& S$ are a mandatory part of the Siegen international graduate course in mechatronics. M\&S is scheduled in the third semester of the mechatronics course and thus these students already have good knowledge of programming and control engineering.

4. A combination of engineering and economics can be studied at Siegen. It is quite probable that these students in their future industrial career will have to decide on the initiation of simulation projects from an economical viewpoint. However they have only very restricted mathematical skills from the undergraduate courses whereas their knowledge of physics is sufficient.

5. Finally, it is also possible for the students from other departments to participate in the courses. However only physicists and mathematicians with basic physics courses have good preconditions for a course on M\&S. Only very engaged students with less preconditions are able to follow the lecture.

\subsection{Lectures}

The lectures on M\&S are currently attended by about 50 students per semester. The course has been broken down to a series of 7 short half semester lectures with four hours per week. Part of the lectures is directly done in the computer laboratory. The courses have already been described in more detail in [2]. From these 7 lectures the different students can choose those units that best fit their requirements and skills. In each case the first course on M\&S is mandatory. This first part is also the subject of the present contribution. Whereas the lectures 2-6 are more advanced with respect to the mathematical and computer science demands the first one is really a CSE course with (almost) no mathematics.

The Siegen courses on M\&S are embedded in the activities of the FOMAAS research center. Founded in 1992 it is concerned with CSE with a special emphasis on the optimization of complex systems. More details about FOMAAS can be taken from 43. Under the auspices of FOMAAS several other lectures can be chosen by the students which complement the basic CSE education. They are dealing for example with finite elements, computational fluid dynamics, electro magnetic field computation, optimization or distributed software systems.

\section{A First Course in Modeling and Simulation}

\subsection{Goals}

The course on 'Foundations of M\&S' has the following goals:

1. To present an overview of the applications of M\&S in academia and industry and to explain the different aims and preconditions of simulation projects. 
2. To establish a first understanding on the general course of a simulation project (see Fig. 1). In particular a rough impression of the financial and manpower efforts of a simulation project is given.

3. An overview on the major methods and tools to carry out continuous time simulations based on ordinary differential equations is given. In particular the students get a survey on some commercially available simulation tools.

4. The basic principles of stochastic simulation are presented. The difference between stochastic and deterministic simulation with respect to accuracy and computing time is emphasized.

5. A general introduction into discrete event simulation which is usually combined with stochastics is given and a survey of major tools is done.

\subsection{How Much Math Is Required?}

With respect to the mathematical prerequisites some compromises must be made in a first course because only two hours in this semester are available:

1. The numerical solution of ODEs is introduced with the Euler algorithm which is easy to understand for the whole audience. Although no specialist will use this algorithm in practice it can well be used to explain many basic problems of numerical ODE solution like accuracy, step size control or instability. More details on the numerical ODE solution are postponed to the second part of the lecture which gives an in depth treatment of this topic. Instead, the preconfigured ODE solvers from MATLAB and SIMULINK are used to demonstrate that they are superior to the Euler method and that it is worthwhile to develop specialized algorithms for certain problems.

2. Although this is a major part of any CSE program no introduction to spatially distributed systems and PDE solution is given. The reason is that even a basic introduction to this domain will take a whole courses' time. Nevertheless, if more time is available then some basics on PDEs can be integrated into the course. The emphasis here should be on a prototypical solution algorithm like finite differences and a discussion of the computational complexity that is associated with PDE problems. Then a commercial finite element tool like FEMLAB could be used to demonstrate the basic steps in the solution of a PDE problem. In fact this is done in the third part of the lectures at the University of Siegen. More details on these subjects are given in the acompanying lectures (see Section 2.2).

3. Typically, students from the engineering departments have little knowledge of probability theory and statistics. Because this is required for stochastic simulation a 'survival kit' in probability and statistics is integrated in the course. This kit contains a basic knowledge of distributions, statistical estimates and tests. The emphasis is not on a broad coverage of topics but on some fundamental results concerning the precision and convergence behavior of stochastic algorithms.

4. What integrators are for ODE solution that are random number generators for stochastic simulation. However, only the basic congruence generator is 
presented as a prototype for more advanced modern generators. The basic problems of pseudo random numbers are shortly discussed. Finally, the most basic methods for the generation of non uniform distributions (transformation, rejection) are presented.

\section{The Looping Swing: An Instructive Example}

As a basic example of a complete simulation project the looping swing is now discussed in more detail. This example requires only little preliminary knowledge from mathematics and physics. It is an important part of the first course on M\&S and takes about ten hours including two hours of computer exercises. It must be pointed out that this example does not reduce a simulation project just to the process of solving a given differential equation system with numerical methods (this is just one of many steps in Fig. 1) but tries to show the course of a simulation project in a realistic application context. The typical steps of a simulation project as shown in Fig. 1 are discussed in the lecture. The following remarks concentrate on the educational and didactic aspects of the looping swing example:

Project specification: The project goal is to find out if it possible to construct a fairground swing that can be driven around the top in a $360^{\circ}$ loop (Fig. 2). In order to make such an apparatus profitable for the operator it should not take too much time until the first looping is managed by an average person. Moreover, for economic reasons the operator must know a suitable initial swing velocity that is required to keep the total swinging time within a reasonable limit. From an educational viewpoint it is very important to emphasize that an operator of a fairground swing is typically not an academically skilled person and thus the communication with this client will be rather difficult for a computational scientist. This should give an impression to the students of how important the initial project

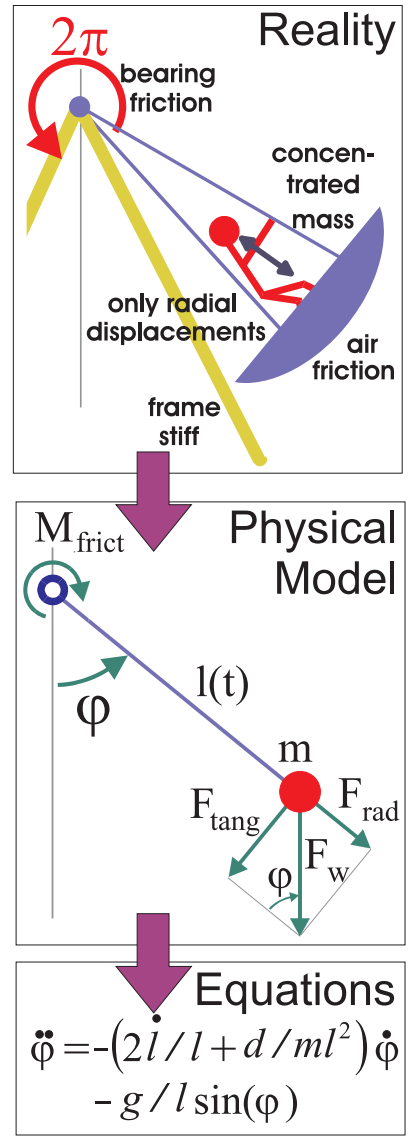

Fig. 2. Modeling of the looping swing: From the real system to the mathematical model specification is for the overall progress.

Mathematical modeling: By common definition simulation must always been based on a mathematical model of the system under investigation. Modeling is usually subdivided into a first step of building an idealized physical representation of the real system and a second step of generating the mathematical equations from this system. As pointed out in 2] these two steps should be 
sharply separated in a lecture. In the running example one major educational goal is to demonstrate the dependency of model complexity on the aim of the simulation project. In this case it is not necessary to predict the dynamics of the looping swing with a high precision. For this reason a very simple representation of the complex system can be used. After a thorough discussion the whole system is reduced to a mathematical pendulum with a time dependent length. Here the important concept of the scope of a mathematical model can be clearly demonstrated. Due to the very simple model (Fig. 2) only the radial movements of the person in the swing are taken into account while tangential movements are neglected. This means that the model is not able to describe the acceleration of the swing starting with an initial angle of zero and zero velocity. Having drawn a picture of the idealized physical model the generation of the mathematical equations is straight forward from the law of angular momentum (Fig. 2).

Implementation: To implement a simulator on a very basic level the Euler method is quickly introduced by deriving it from a differential quotient approximation of the time derivative. The well-known deficiencies of this primitive ODE solver are rather an advantage in a first course on simulation than a disadvantage because the shortcomings of the Euler method will immediately turn out in the next steps of the project. In the next unit of the lecture which is concerned with tools for continuous time simulation the Euler method is compared with other integrators contained in the MATLAB and SIMULINK packages. This in turn motivates to learn more about numerical algorithms in the subsequent lectures. Data acquisition: It is frequently disregarded that the acquisition of data to parameterize a model can be one of the most time consuming parts of a simulation project in practice. This can be illustrated even with the simple swing model. It is discussed how empirical data, material constants, separate calculations, data sheets of component manufactures, parameters from comparable systems, interviews with the operator, or experiments can be used as data sources in the present example.

Verification: The term verification means to prove the correctness of the simulator implementation relative to the idealized physical model. In the example the plausibility of the model is tested by doing extensive parameter and initial value variation studies. In each case it is checked if the model behaves in the expected way. Because the mathematical pendulum can be approximated for small angles by a linear model an analytical solution is available. This solution can also be taken to check the correctness of the implementation.

Validation: The term model validation means to prove the correct representation of the real system by the simulation with respect to the accuracy requirements and the experimental frame given in the project specification. Model validation is another very critical step in a simulation project which is closely related to the very fundamentals of natural science [7]. Two aspects of model validation are considered in the example. The first aspect is the friction model 
contained in the system equations. By comparing different friction models (constant, linear, quadratic, mixed) it turns out that there is a difference but this difference is not important for the problem solution. Thus a linear friction model can be safely assumed although it might not exactly represent the reality. The friction coefficient must be chosen with a reasonable order of magnitude. The second aspect of validation is the estimation of model parameters which could not be obtained from available data. Two methods to estimate the linear friction coefficient from experiments are shortly discussed which are the direct usage of the analytical solution or general parameter fitting procedure.

Problem solution: In reality swings are accelerated by the same mechanism that is used in iceskating to perform a pirouette. To increase the rotational velocity the center of mass has to be moved closer to the center of rotation. Unlike in iceskating this cannot be continuously done in a looping swing. However, the swing length can be increased without an effect on the rotational energy at the turning points of the swing. This is also the recipe to build up a control strategy for the looping swing. Interestingly, this strategy is not that easy to implement because further restrictions due to human limitations like smoothness and bounded accelerations must be recognized. Moreover, the control strategy should account for the 'sportiveness' of the swing driver. A parametrized analytical control function is constructed based on piecewise scaled trigonometric functions. Simulation experiments can now be carried out to solve the original problem. It turns out that a well trained driver is able to achieve a looping after 15 swings.

Result presentation: The presentation of the results of a simulation study is another very important step in practice because they must be communicated to a customer who may not be familiar with the basics of simulation. Thus it is very important which graphical visualisation is used to demonstrate the results. To this end several different visualizations for the dynamics of the looping swing are introduced and compared with respect to their possible audience (Fig. 3). Clearly, the virtual rea-
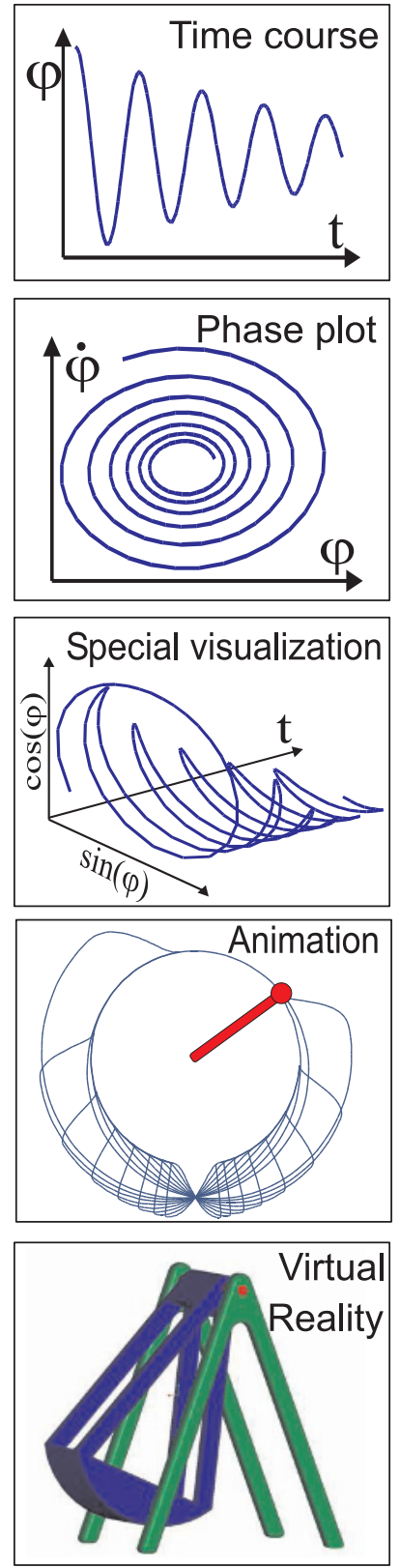

Fig. 3. Different visual representations of the simulation results 
lity representation (bottom) is totally 'oversized' because this is essentially a 2-dimensional system. Nevertheless it will be the most convincing visualisation for an operator of a fairground swing.

Application: In both academic and industrial applications simulation projects have the tendency to be never finished because once the results have been presented and applied to the real system new problems will arise. In the case of the boat swing this might be the problem of how to safely fix the swing driver to the swing body. In many situations it may be the case that some important details of the real system have been disregarded. In the present example it may be the case that the swing representation by the mathematical pendulum is too much simplified and a more detailed physical pendulum model has to be taken.

\section{Experiences}

In the first years of the introductory course on the 'Foundations of M\&S' about 100 students with very different scientific backgrounds have attended it. The chosen mathematical contents are well accepted and - as the first exams and project works show - can be applied by the students in practice. By conception the students have the opportunity to choose any other course of the series M\&S 2-7 afterwards. In fact many students with a sound mathematical background (like the mechatronics students) participate in 3-4 courses while the others rather choose one of the more practical courses like 'M\&S 7: Simulation tools'. The Siegen concept of a modular education in computational science for students from different disciplines will be extended and more institutionalized in the next years by installing CSE as a specialization that can be chosen in different courses like engineering, computer science or mathematics.

\section{References}

1. W. Wiechert, Lecture Notes on Modeling and Simulation, http://www.simtec.mb.uni-siegen.de.

2. W. Wiechert, W., The Role of Modeling in Computational Science Education. Future Generation Computer Systems, 2003, pp.1363-1374.

3. FOMAAS (Forschungszentrum für Multidisziplinäre Analysen und Angewandte Systemoptimierung): University of Siegen, http://www.fomaas.uni-siegen.de.

4. W. Wiechert, W. Becker, H.A. Eschenauer, B. Freisleben, M. Grauer, D. Hartmann, H.-J. Reinhardt, and G. Thierauf, eds. FOMAAS Status-Report 2000-2002. 2002. ISBN 3-9807356-3-x

5. W. Wiechert, Eine Checkliste für den Aufbau einer Simulationstechnik Vorlesung, in: K. Panreck, F. Dörrscheid, 15. Symposium Simulationstechnik, Paderborn, SCS Publishing House, 2001, pp. 151-156.

6. B.F. Gore, The Child's Swing. Journal of Physics, 1970. 38: p. 378-379.

7. W. Wiechert, R. Takors, Validation of metabolic models: Concepts, tools, and problems, in: H.V. Westerhoff, B.N. Kholodenko (Eds.), Metabolic Engineering in a Post Genomic Era, Horizon Scientific Press, 2003. 